\begin{tabular}{|c|l|}
\hline Title & Reduced interface reaction during the epitaxial Fe growth on InA s for high efficiency spin injection \\
\hline Author(s) & Yoh, Kanji; Ohno, Hiroshi; Sueoka, Kazuhisa; Ramsteiner, Manfred E. \\
\hline Citation & $\begin{array}{l}\text { Journal of V acuum Science \& Technology B: Microelectronics and Nanometer Structures, 22(3), 1432-1435 } \\
\text { https:/doi.org/10.1116/1.1755711 }\end{array}$ \\
\hline Issue Date & 200405 \\
\hline Doc URL & http://hdl.handle.net/2115/28637 \\
\hline Rights & ○2004 A merican V acuum Society \\
\hline Type & article \\
\hline File Information & JV STB22-3.pdf \\
\hline
\end{tabular}

Instructions for use 


\title{
Reduced interface reaction during the epitaxial Fe growth on InAs for high efficiency spin injection
}

\author{
Kanji Yoh ${ }^{\mathrm{a})}$ \\ Research Center for Integrated Quantum Electronics, Hokkaido University, Sapporo 060-8628, Japan \\ and CREST-JST (Japan Science and Technology Agency), Japan \\ Hiroshi Ohno and Kazuhisa Sueoka \\ Graduate School of Engineering, Hokkaido University, Sapporo 060-8628, Japan \\ Manfred E. Ramsteiner \\ Paul Drude Institute of Solid State Electronics, Berlin 060-8628, Germany
}

(Received 27 October 2003; accepted 5 April 2004; published 7 June 2004)

\begin{abstract}
We have investigated Fe/InAs interfaces for two different growth temperatures of $\mathrm{Fe}$ and the effect on the spin injection properties through an Fe/InAs junction. Secondary ion mass spectroscopy and transmission electron microscopy studies of the Fe/InAs interfaces revealed that Fe films grown at $175^{\circ} \mathrm{C}$ clearly suffer from increased reaction and out-diffusion of semiconductor constituents compared to those grown at $23{ }^{\circ} \mathrm{C}$. The lower temperature samples showed an increased degree of spin polarization of $18 \%-20 \%$ which translates to $36 \%-40 \%$ of spin injection efficiency assuming selection rules between heavy and light holes in the $p$-type InAs substrate. It is close to the spin polarization of 40\%-45\% in an Fe spin injector itself. (C) 2004 American Vacuum Society.
\end{abstract}

[DOI: $10.1116 / 1.1755711]$

\section{INTRODUCTION}

Spin injection from ferromagnetic metals into semiconductors is one of the key technologies to achieve spintronics ${ }^{1}$ in the future. In spite of its potential importance, there have been keen discussions on whether or not efficient spin injection into a semiconductor is possible at all. Experimentally, spin injection from high $T_{c}$ ferromagnetic metals into semiconductor has been reported by several groups ${ }^{2}$ in the $\mathrm{Fe} /$ GaAs system where electrons tunnel through a Schottky barrier which would drastically limit the drain current. Besides, those tunneling barriers, which might work as a spin filter, are believed to be the very element that enables spin injection through a ferromagnet/semiconductor junction. We have chosen InAs as a semiconductor material because $\mathrm{Fe}(100)$ on $p$-InAs(100) is expected to form an ideal ohmic contact ${ }^{3,4}$ with no Schottky barrier. In addition, a more significant spinorbit interaction is expected in an InAs two-dimensional electron gas channel ${ }^{5,6}$ than in GaAs, which will be beneficial to achieve a spin transistor in the future. The purpose of the present work is to show that the control of the interface reaction between $\mathrm{Fe}$ and InAs plays an important role in achieving efficient spin injection and eventually to show that there is not a fundamental $\operatorname{limit}^{7,8}$ for spin injection nor the necessity of a tunneling barrier for appreciable spin injection. ${ }^{9}$ We will show how the growth temperature affects interface quality and spin injection efficiency. Finally, high efficiency spin injection is demonstrated. For this purpose, we have epitaxially grown $\mathrm{Fe}$ on InAs at two different temperatures, i.e., 23 and $175^{\circ} \mathrm{C}$. The interface reaction and cross diffusion have been investigated by secondary ion mass spectroscopy (SIMS) analysis and transmission electron mi-

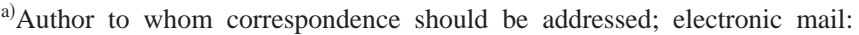
kanjiyoh@aol.com
}

croscopy (TEM) observation. The degree of spin injection efficiency was evaluated by circular polarization measurement of electroluminescence (EL) emission from the $\mathrm{Fe} / p$-InAs sample grown at $23^{\circ} \mathrm{C}$.

\section{FABRICATION AND MEASUREMENT SETUP}

High crystal quality bcc Fe thin films were grown on zincblende InAs wafer in a ultrahigh vacuum at different temperatures. The substrates were thermally cleaned in vacuum without As overpressure prior to the Fe irradiation. Fe was deposited using an e-beam evaporator EFM3(Omicron). In the initial studies of $\mathrm{Fe}$ growth on semiconductors, especially on GaAs, growth temperatures of $150-175^{\circ} \mathrm{C}$ have been preferred $^{10,11}$ probably due to better growth of the $\mathrm{Fe}$ film itself. However, when it comes to the optical and electrical characteristics of the Fe/InAs heteroepitaxial material system, this temperature range did not necessarily yield the best result based on our initial study of this system. ${ }^{4}$ The two growth temperatures chosen for the present study of the growth of $\mathrm{Fe}$ on InAs were 23 and $175^{\circ} \mathrm{C}$. Both samples were subjected to surface and interface analysis by reflection high energy electron diffraction (RHEED) during growth and by SIMS and TEM analysis after growth.

Then, the Fe films were patterned into line/space array (width: $w=2 \mu \mathrm{m}$, pitch: $p=4 \mu \mathrm{m}$ ) so that circularly polarized light could be observed at the top of the sample in parallel with the external magnetic field. EL was detected from the top of the sample by an InSb photodetector. For polarization measurements, the $\sigma^{+}$and $\sigma^{-}$components of the luminescence were measured by switching the angle of a quarter-wave plate. The spin polarization, $P_{S}$, of injected electrons can be estimated from the degree of circular polarization $P_{L}$ of the luminescence due to the optical selection 

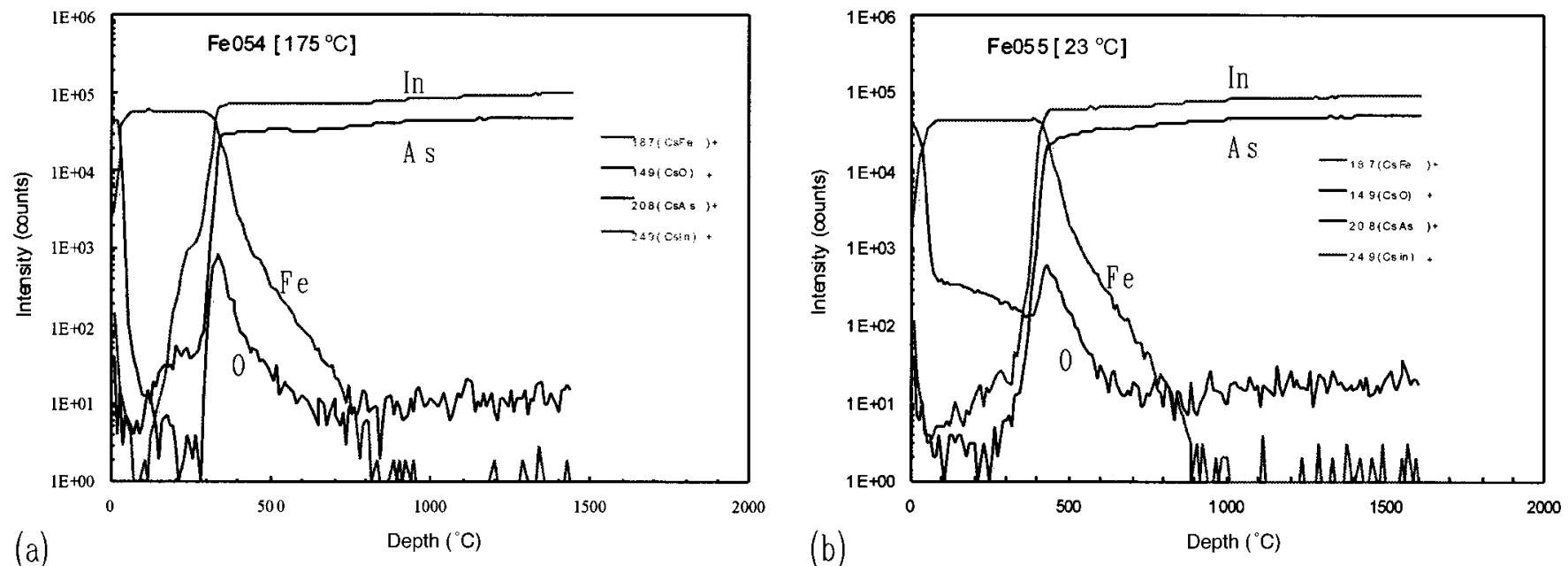

FIG. 1. SIMS profile of Fe/InAs samples grown at (a) $175^{\circ} \mathrm{C}$ and (b) $23{ }^{\circ} \mathrm{C}$. Increased out-diffusion of In and As in Fe layer is observed in (a) $175{ }^{\circ} \mathrm{C}$ sample.

rules in zinc blende compound semiconductors. ${ }^{12}$ In a condition of constant carrier generation, such as constant bias voltage, $P_{L}$ is given by

$$
P_{L}=-\frac{1}{2} \frac{1}{\left(1+2 \tau_{\mathrm{rec}} / \tau_{s}\right)} P_{S},
$$

where $\tau_{\text {rec }}$ and $\tau_{s}$ are the recombination time and the spin relaxation time of electrons, respectively.

Note that the polarization of the luminescence is reduced from the spin polarization of electrons by the factor $\tau_{\text {rec }} / \tau_{s}$. However, limited data are reported for the carrier dynamics and spin dynamics in bulk InAs so far. The spin relaxation in bulk InAs was reported by Boggess et al. ${ }^{13}$ According to their result, $\tau_{\text {rec }}$ and $\tau_{s}$ at $300 \mathrm{~K}$ are 1 and $20 \mathrm{ps,} \mathrm{respectively.}$ If we assume that $\tau_{\text {rec }} \ll \tau_{s}$ even at low temperatures, the factor $1 /\left(1+2 \tau_{\text {rec }} / \tau_{s}\right)$ becomes almost negligible. In that case, we obtain a simple factor of $-1 / 2$ (i.e., $P_{S}=-2 P_{L}$ ). In general, the temperature dependence of the $P_{L}$ would be determined by the temperature dependence of these time constants. It is to be noted that the discussion above assumes that the heavy hole and light hole bands are degenerate in InAs.

\section{EXPERIMENTAL RESULTS AND DISCUSSIONS}

RHEED observations revealed that low temperature $\left(23^{\circ} \mathrm{C}\right)$ growth yields three-dimensional-like growth in the initial stage compared with the high temperature $\left(175^{\circ} \mathrm{C}\right)$ growth sample. ${ }^{4}$ However, smooth two-dimensional growth is recovered in both cases after $400 \AA$ of Fe growth. This is followed by $30 \AA$ Au cap layer. ${ }^{4}$ On the other hand, SIMS measurement revealed that the Fe layer grown at the higher temperature contained more As and In due to diffusion as seen in Fig. 1. This result suggests that lower temperature growth is preferred to reduce possible interface reactions while high temperature growth seems to be preferred to obtain better crystal quality of Fe films at least in the initial stage. The Fe and oxygen tails that extend from the interface into the InAs substrate (Fig. 1) are presumably artifacts of the SIMS measurement. The existence of oxygen at the interface in both samples comes from residual oxygen adsorbed during the exposure of the sample to air prior to the Fe growth. Note that the oxygen level at the interface is two orders of magnitude less than the surface oxygen level at the topmost thin layer of about $50 \AA$ where thin iron oxide is formed. It is difficult to make conclusions about the thickness and composition of possible compound formation at the interface in both cases because of the depth resolution of SIMS analysis.

However, it is clear that the sample grown at $175^{\circ} \mathrm{C}$ suffers from increased interdiffusion at the interface compared with the one grown at the lower temperature $\left(23^{\circ} \mathrm{C}\right)$. It is remarkable to observe such a clear difference in interface structures with only $150{ }^{\circ} \mathrm{C}$ difference in growth temperature. In order to verify possible reactions in a more microscopic way, we have conducted TEM image analysis on the same samples used for SIMS analysis. Overall, clean interfaces are observed in both cases. In the large area image (Fig. 2) a thin white region in the $175^{\circ} \mathrm{C}$ sample is observed which may suggest the existence of some thin interface layer formation on, whereas in the $23^{\circ} \mathrm{C}$ sample image no such thin white region occurs at the interface. However, the possibility of an artifact cannot be completely excluded at this point. The uneven dark/bright pattern in the InAs substrate side near the interface seems to be more pronounced in the $23{ }^{\circ} \mathrm{C}$ sample, suggesting pronounced interface strain in this sample. These observations may be indicating possible interface layer formation in the $175^{\circ} \mathrm{C}$ sample which accommodates strain between the InAs substrate and the Fe layer. Clearly these strain-induced patterns on the semiconductor side show different pitches; the $23^{\circ} \mathrm{C}$ sample shows a shorter pitch than the $175^{\circ} \mathrm{C}$ sample, indicating smaller grain size in the former. We have looked at the interface in higher magnification to find some evidence on the hypothesis. Figure 3 shows high resolution TEM patterns on both samples. A few monolayers (less than ten) of interface substance can be seen in the $175^{\circ} \mathrm{C}$ sample, whereas a more abrupt interface is seen in the $23{ }^{\circ} \mathrm{C}$ sample. These blow-up pictures were taken from 


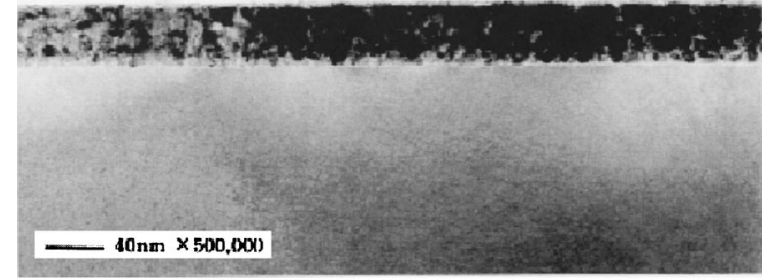

(a) Tsub $=175 \mathrm{C}$

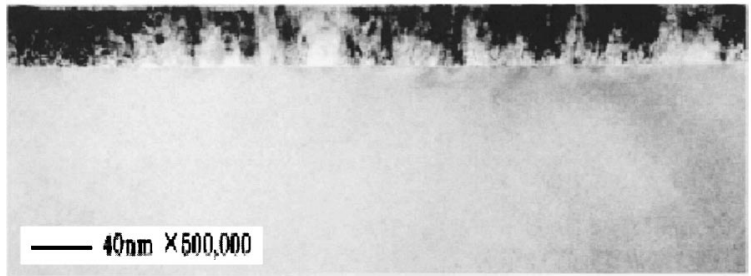

(b) $\mathrm{Tsub}=23 \mathrm{C}$

FIG. 2. TEM image analysis (1). About $400 \AA$ of Fe film is seen to grow on InAs substrate. Above sample (a) shows Fe growth at $T_{\text {sub }}=175^{\circ} \mathrm{C}$, and the bottom sample (b) $23{ }^{\circ} \mathrm{C}$.

each sample at random. It seems to be consistent with the SIMS data that more interface reaction takes place at $175^{\circ} \mathrm{C}$ than at $23^{\circ} \mathrm{C}$. In addition to the relative difference, the $23^{\circ} \mathrm{C}$ sample does not seem to suffer from appreciable interface reaction from both SIMS and TEM image analysis. Those reactions must become significant at temperatures greater than $23{ }^{\circ} \mathrm{C}$. In the next paragraph, circular polarization measurement will be briefly discussed using the sample grown at $23{ }^{\circ} \mathrm{C}$.

Spin polarization of the $\mathrm{Fe} / \mathrm{InAs}$ sample grown at $23^{\circ} \mathrm{C}$ was characterized as shown schematically in Fig. 4. A clear electroluminescence (EL) signal was detected from the top of the sample with an InSb photodetector. The EL data showed clear spin injection when compared with the EL of nonmagnetic samples and photoluminescence of InAs substrate despite the theoretical controversy ${ }^{3,4}$ on spin injection. The observed degree of circular polarization was estimated to be $\sim 12 \%$. $^{5}$ The $\sigma^{+}$and $\sigma^{-}$components of the EL were measured by switching the angle of the quarter-wave plate. Figure 5 shows the external magnetic field dependence of the

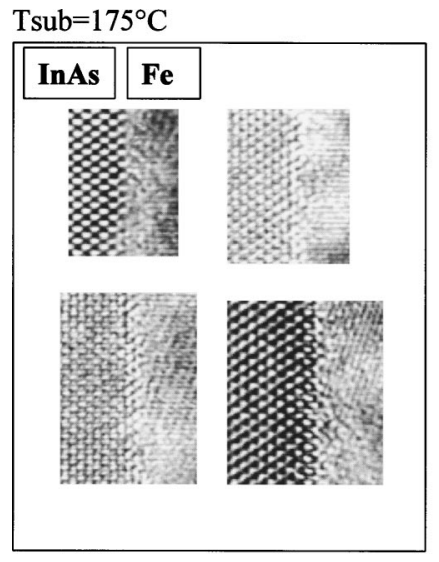

Tsub $=23^{\circ} \mathrm{C}$

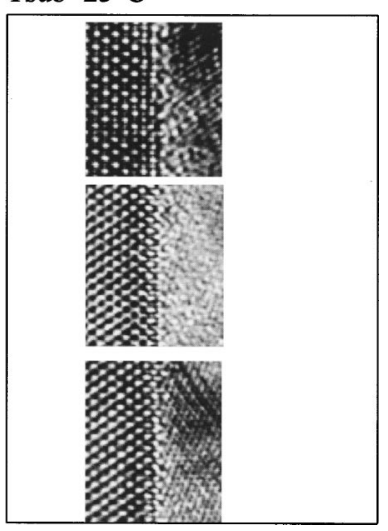

FIG. 3. TEM image analysis (2). Blow-up TEM image of both samples.

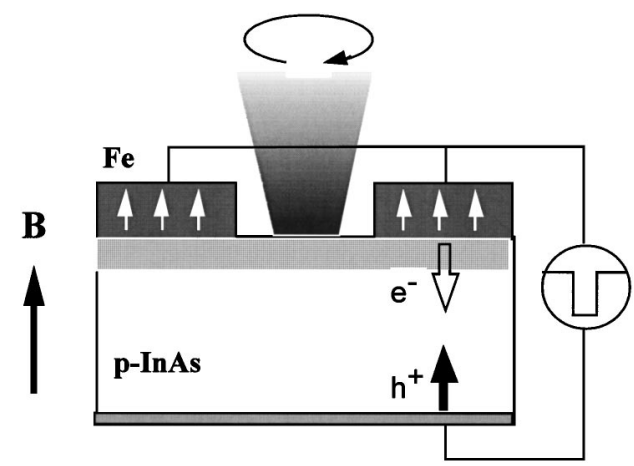

FIG. 4. Schematic cross-sectional diagram of the EL sample. Electroluminescent light is detected at the top of the sample by InSb detector.

polarization efficiency calculated from the measured intensities of the $\sigma^{+}$and $\sigma^{-}$components of the luminescence $P_{L}$. Considering the opposite polarization behavior from the nonmagnetic sample (EL) and the bare InAs substrate $(P l)$, which is due to the Zeeman effect, the net polarization in the Fe electrode is estimated to be much higher, approaching $18 \%-20 \%$. Based on this degree of circular polarization result, the corresponding spin injection efficiency is calculated to be $36 \%-40 \%$ as illustrated in Fig. 6. This calculation is based upon the assumption that the spin relaxation time is much longer than the energy relaxation time and the degeneracy of the heavy and light hole bands in the $p$-InAs substrate. This spin injection efficiency result compares favorably with the spin polarization measurement of $40 \%-45 \%$ in Fe. ${ }^{14}$ Because our interface study shows that the Fe/ $p$-InAs system contains no appreciable tunnel barrier on the atomic resolution scale, it can be concluded that the spin injection occurs with such a high efficiency even without the existence of tunnel barriers. This is contradictory to the so-called "conductivity mismatch" discussion in the diffusion theory, ${ }^{7,8}$ but agrees with first principle calculations. ${ }^{15,16}$

\section{CONCLUSION}

We have investigated the Fe/InAs interface for two different growth temperatures of $\mathrm{Fe}$ and its effect on the interface structure of the Fe/InAs junction. Secondary ion mass spectroscopy (SIMS) and transmission electron microscopy (TEM) studies of the Fe/InAs interfaces revealed that the

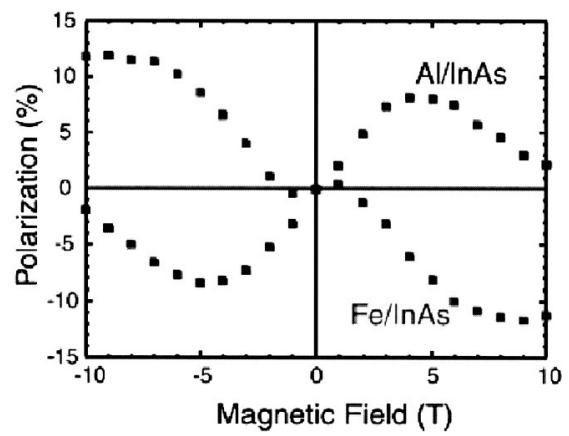

FIG. 5. Degree of circular polarization of EL from Al/InAs and Fe/InAs structures. 


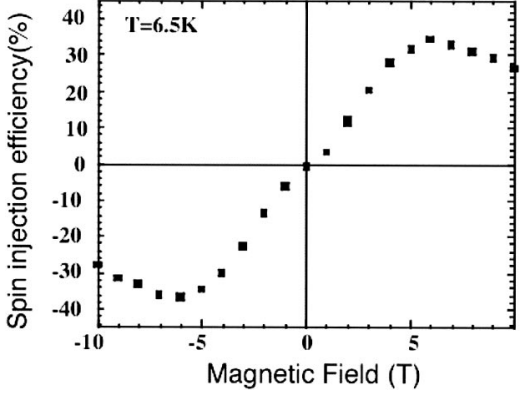

FIG. 6. Net spin injection efficiency in an Fe/InAs structure.

samples grown at $175^{\circ} \mathrm{C}$ clearly suffer from increased reaction and out-diffusion of semiconductor constituents compared with that of $23^{\circ} \mathrm{C}$ grown samples. The lower temperature sample showed an $18 \%-20 \%$ degree of spin polarization which translates to $36 \%-40 \%$ of spin injection efficiency assuming selection rules between heavy and light holes in the $p$-type InAs substrate. The present result is close to the spin polarization of $40 \%-45 \%$ on $\mathrm{Fe}$ spin injector itself.

\section{ACKNOWLEDGMENTS}

The authors thank Dr. A. Kawaharazuka for his technical support during the EL measurement with vertical magnetic field. They are also grateful to Professor K. Ploog of the Paul Drude Institute for the technical discussions. This work was partially supported by Grant-in-Aid for Scientific Research from the Ministry of Culture, Sports, Science and Technology. The authors also acknowledge the partial support from CREST (Japan Science and Technology Agency).

${ }^{1}$ S. Datta and B. Das, Appl. Phys. Lett. 56, 665 (1990).

${ }^{2}$ H. J. Zhu, M. Ramsteiner, H. Kostial, M. Wassermeier, H.-P. Schonherr, and K. H. Ploog, Phys. Rev. Lett. 87, 016601 (2001).

${ }^{3}$ Y. B. Xu, E. T. M. Kernohan, M. Tselepi, J. A. C. Bland, and S. Holmes, Appl. Phys. Lett. 73, 399 (1998).

${ }^{4}$ H. Ohno, K. Yoh, T. Doi, A. Subagyo, K. Sueoka, and K. Mukasa, J. Vac. Sci. Technol. B 19, 2280 (2001).

${ }^{5}$ J. Luo, H. Munekata, F. F. Fang, and P. J. Stiles, Phys. Rev. B 38, 10142 (1988).

${ }^{6}$ E. A. de Andrada e Silva, G. C. La Rocca, and F. Bassani, Phys. Rev. B 50, 8523 (1994).

${ }^{7}$ P. C. van Son, H. van Kempen, and P. Wyder, Phys. Rev. Lett. 58, 2271 (1987).

${ }^{8}$ G. Schmidt, D. Ferrand, L. W. Molenkamp, A. T. Filip, and B. J. van Wees, Phys. Rev. B 62, R4790 (2000).

${ }^{9}$ E. I. Rashba, Phys. Rev. B 62, R16267 (2000)

${ }^{10}$ C. Daboo, R. J. Hicken, E. Gu, M. Gester, S. J. Gray, D. E. P. Eley, E. Ahmed, and J. A. C. Bland, Phys. Rev. B 51, 15964 (1995).

${ }^{11}$ Y. B. Xu, E. T. M. Kernohan, M. Tselepi, J. A. C. Bland, and S. Holms, Appl. Phys. Lett. 73, 399 (1998).

${ }^{12}$ M. I. Dyakonov and V. I. Perel, Optical Orientation, edited by F. Meier and B. P. Zakharchenya (Elsevier, North-Holland, Amsterdam, 1984), Chap. 2, pp. 11-71.

${ }^{13}$ T. F. Boggess, J. T. Olesberg, C. Yu, M. E. Flatte, and W. H. Lau, Appl. Phys. Lett. 77, 1333 (2000).

${ }^{14}$ R. J. Soulen, Jr. et al., Science 282, 85 (1998).

${ }^{15} \mathrm{O}$. Wunnicke, Ph. Mavropoulos, R. Zeller, and P. H. Dederichs, Phys. Rev. B 65, 241306(R) (2002).

${ }^{16}$ M. Zwierzycki, K. Xia, P. J. Kelly, G. E. W. Bauer, and I. Turek, arXiv:cond-mat/0204422 (2002). 